\title{
PROPRIEDADES FÍSICAS DE UM LATOSSOLO VERMELHO-AMARELO CULTIVADO COM CAFEEIRO EM TRÊS SISTEMAS DE MANEJO NO SUL DE MINAS GERAIS ${ }^{(1)}$
}

\author{
Davi Lopes do Carmo ${ }^{(2)}$, Dulcimara Carvalho Nannetti ${ }^{(3)}$, Moacir de \\ Souza Dias Júnior ${ }^{(4)}$, Djalma José do Espírito Santo ${ }^{(5)}$, Alex \\ Nogueira Nannetti ${ }^{(6)}$ \& Tales Machado Lacerda ${ }^{(5)}$
}

\begin{abstract}
RESUMO
Em razão de diferentes tipos de manejo utilizados na cafeicultura, o presente trabalho teve como objetivo quantificar as alterações de alguns atributos físicos de um Latossolo Vermelho-Amarelo cultivado com cafeeiro, após quatro anos da implantação, no Sul de Minas Gerais, sob três sistemas de manejo em comparação à mata nativa. Foram avaliados os seguintes sistemas de manejo: lavoura cafeeira com mecanização (CCM), lavoura cafeeira sem mecanização (CSM), lavoura cafeeira sob sistema adensado (CA) e mata nativa (MN), utilizada como referência. Foram coletadas amostras indeformadas, com o auxílio de um amostrador de Uhland e anéis de alumínio de $6,35 \mathrm{~cm}$ de diâmetro por $2,54 \mathrm{~cm}$ de altura, nas profundidades de 0-3 e 15-18 cm, sendo esta última a camada de máxima resistência mecânica determinada previamente por penetrometria. As amostras foram retiradas em duas posições nas lavouras cafeeiras, sendo na linha de tráfego das máquinas e na projeção da copa dos cafeeiros, nos manejos CCM e CSM; no centro das entrelinhas e na projeção da copa do cafeeiro, no manejo CA; e aleatório, na mata nativa, com quatro repetições, totalizando 56 amostras. Os atributos físicos avaliados foram densidade do solo (Ds), densidade de partícula (Dp), resistência
\end{abstract}

\footnotetext{
(1) Projeto desenvolvido em parceria do Instituto Federal de Educação, Ciência e Tecnologia do Sul de Minas Gerais - Campus Machado-MG (IF - Campus Machado) e o Departamento de Ciência do Solo da Universidade Federal de Lavras - DCS/UFLA. Recebido para publicação em fevereiro de 2010 e aprovado em março de 2011.

(2) Mestrando em Ciência do Solo pela Universidade Federal de Lavras - UFLA Caixa Postal 3037, CEP 37200-000 Lavras (MG). E-mail: davigoldan@yahoo.com.br

(3) Professores do IF - Campus Machado-MG, rodovia Machado-Paraguaçu, km 07, CEP 37750-000 Machado (MG). E-mail: dulcimara@mch.ifsuldeminas.edu.br

(4) Professor Associado do Departamento de Ciência do Solo da Universidade Federal de Lavras - UFLA Caixa Postal 3037, CEP 37200-000 Lavras (MG). Bolsista CNPq e Pesquisador Mineiro-FAPEMIG. E-mail: msouzadj@ufla.br

(5) Tecnólogos em Cafeicultura pelo IF - Campus Machado-MG. E-mails: tales_lacerda@yahoo.com.br; dj.santo@uol.com.br

(6) Engenheiro Agrônomo - Mestrando em Produção na Agropecuária - UNIFENAS-MG.
} 
do solo à penetração (RP), volume total de poros (VTP), macroporosidade (Ma), microporosidade (Mi) e relação $\mathrm{Ma} / \mathrm{Mi}$. O sistema de café com mecanização alterou as propriedades físicas, na posição de linha de tráfego de máquinas, indicadas pelo aumento da densidade do solo e resistência do solo à penetração e pela redução do volume total de poros, macroporosidade e da relação macro/microporosidade, quatro anos após o plantio. Comparando as profundidades, o sistema de café com mecanização apresentou menores valores de macroporosidade e relação macro/ microposidade e maiores valores de microporosidade e resistência do solo à penetração, na profundidade de 0-3 cm, quatro anos após o plantio.

Termos de indexação: estrutura do solo, sustentabilidade, mecanização.

\title{
SUMMARY: PHYSICAL PROPERTIES OF A RED-YELLOWLATOSOL UNDER THREE DIFFERENT SYSTEMS OF COFFEE MANAGEMENT IN THE STATE OF MINAS GERAIS
}

\begin{abstract}
In view of the different managements used in coffee production, this study aimed to quantify the changes of some physical properties of an Oxisol under four-year-old coffee, in southern Minas Gerais. Three management systems were compared to native forest. The following management systems were evaluated: mechanized coffee production (CCM), coffee without mechanization (CSM), dense coffee plantation (CA) and native forest (MN) as reference. Undisturbed samples were collected with an Uhland sampler and aluminum rings (diameter $6.35 \mathrm{~cm}$, height $2.54 \mathrm{~cm}$ ), from the layers $0-3 \mathrm{~cm}$ and $15-18 \mathrm{~cm}$; in the latter the maximum mechanical resistance had been previously determined by a penetrometer. The coffee plantations were sampled at two points, i.e., in the wheel track and under the trees (canopy projection) under CCM and CSM management, in-between coffee rows and under the trees under CA management, and randomly in native forest, with four replications, totaling 56 samples. The physical properties soil bulk density $(B D)$, particle density $(D p)$, soil resistance to penetration $(R P)$, total pore volume (VTP), macroporosity (Ma) and microporosity (Mi) and Ma/Mi ratio were evaluated. The mechanized coffee system affected the physical properties in the wheel track, as indicated by increases in soil density and soil penetration resistance and reduced total porosity, macroporosity and Ma/Mi ratio, four years after planting. In the 0-3 cm layer, macroporosity and Ma/Mi ratio were lower in the mechanized coffee system and microporosity, and penetration resistance higher than in the deeper layer, four years after planting.
\end{abstract}

Index terms: soil structure, sustainability, mechanization.

\section{INTRODUÇÃO}

A produção de café é uma das atividades mais importantes para a economia do Brasil, o maior produtor de café do mundo, totalizando 39.470 milhões de sacas em 2009. Minas Gerais é o Estado de maior produção, com cerca de 19.880 milhões de sacas, o que corresponde aproximadamente a $50 \%$ da produção nacional (Conab, 2009).

No empreendimento da cafeicultura são utilizadas várias práticas de manejo, visando, sobretudo, à busca por resultados positivos na produtividade, na diminuição dos custos, na qualidade do solo e no impacto ambiental. No entanto, essas práticas podem alterar as propriedades físicas do solo, especialmente nas camadas superficiais. $\mathrm{O}$ aumento da população de plantas por unidade de área, ou seja, plantios adensados, tem se destacado devido a aumento de produtividade, aumento da fertilidade do solo (Pavan et al., 1999) e melhoria da agregação das partículas do solo, com implicações diretas na infiltração de água e resistência do solo à erosão (Pavan \& Chaves, 1996). O aumento do número de plantas por área oferece maior proteção ao solo, em razão da maior cobertura proporcionada pelo próprio cafeeiro e do maior número de folhas que caem sobre o solo, diminuindo a erosão, temperatura, lixiviação e volatilização de nutrientes, evaporação da água, além de intensificar a reciclagem de nutrientes no solo.

A mecanização do cafeeiro justifica-se pelo alto custo da mão de obra, em especial na colheita; no entanto, o uso intensivo de máquinas e equipamentos agrícolas em condições inadequadas de umidade do solo pode causar a sua compactação (Dias Junior, 2000; Silva 
et al., 2002). Segundo Silva et al. (2006), a intensidade das operações mecanizadas em lavouras cafeeiras é aumentada na estação chuvosa, condição em que o solo apresenta elevada umidade. A compactação dos solos agrícolas provocada pelo tráfego de máquinas tem sido relatada por vários autores (Silva et al., 1986; Novak et al., 1992; Silva et al., 2003) e identificada pelo aumento da capacidade de suporte de carga do solo, pelo aumento da resistência mecânica do solo e da densidade deste e pela redução na porosidade total (Dias Junior, 2000; Miranda et al., 2003), levando a efeito adverso sobre o crescimento de raízes e consequente diminuição na absorção de água e de nutrientes (Ishaq et al., 2001), o que resulta em decréscimo da produção (Dauda \& Samari, 2002). De acordo com o sistema de manejo do solo utilizado, o grau de compactação depende da intensidade de preparo e da espessura da camada mobilizada, do histórico de uso, de como as pressões são aplicadas, da umidade no momento das operações de cultivo e do número e frequência das passadas dos equipamentos em cada safra (Secco et al., 2004). Em lavouras cafeeiras, a área de trabalho com a mecanização é restrita a faixas pequenas nas entrelinhas de plantio, nas quais as máquinas transitam sempre no mesmo local (Carvalho Filho et al., 2004), e próximo à copa do cafeeiro, onde se concentra parte das raízes absorventes, ficando o crescimento das raízes sujeito às restrições do solo. Com isso, para se obter uma cafeicultura sustentável, é necessária a adoção de técnicas que visam evitar a compactação do solo, como a utilização de modelos de capacidade de suporte de carga, que predizem a máxima pressão que um solo pode suportar em diferentes umidades sem sofrer compactação adicional (Dias Junior, 1994).

De modo geral, o solo sob mata nativa apresenta características físicas adequadas ao crescimento e desenvolvimento normal de plantas (Andreola et al., 2000), por se encontrar sob condição estável (Bayer \& Mielniczuk, 2008). Com isso, atributos de solo oriundos de áreas de mata nativa, para fins de comparação com atributos do solo de lavouras de cafeeiro sob manejo intensivo (Gontijo et al., 2008), têm sido utilizados como indicadores de qualidade do solo. Contudo, é de grande interesse identificar o impacto na estrutura do solo, decorrente da adoção de diferentes sistemas de manejo ao longo dos anos, visando à uma cafeicultura sustentável.

Entre os indicadores físicos de qualidade do solo, Karlen \& Stott (1994) relacionaram densidade do solo, porosidade e estrutura, e Tormena \& Roloff (1996), a resistência mecânica do solo, dentre outros. O crescimento do sistema radicular das plantas pode ser reduzido com aumento da densidade do solo devido ao impedimento físico. De acordo com Torres \& Saraiva (1999), a densidade do solo varia de acordo com as suas características: em solos argilosos, varia de 1,0 a $1,45 \mathrm{Mg} \mathrm{m}^{-3}$ para condições de mata e muito compactado, respectivamente. A resistência do solo à penetração na faixa de 1,5 a 3,0 $\mathrm{MPa}$ é restritiva ao crescimento radicular (Grant \& Lanfond, 1993). Para Arshad et al. (1996), o crescimento de raízes é limitado em solos com valores de resistência à penetração maiores que 2,0 $\mathrm{MPa}$. Os valores críticos de macroporosidade estão abaixo dos valores compreendidos entre 0,10 e 0,16 m $\mathrm{m}^{-3}$ (Baver et al., 1972).

Objetivou-se no presente trabalho quantificar as alterações de alguns atributos físicos de um Latossolo Vermelho-Amarelo cultivado com cafeeiro, após quatro anos da implantação, no Sul de Minas Gerais, sob três sistemas de manejo, em comparação à mata nativa.

\section{MATERIAL E MÉTODOS}

A área de estudo localiza-se na Fazenda Santa Rita, no município de Machado, Sul de Minas Gerais, a uma latitude de $21^{\circ} 35$ ' 19 ", longitude de $45^{\circ} 53$ ' 02 " W de Greenwich e altitude de $984 \mathrm{~m}$. O clima da região é Cwa, segundo a classificação de Köppen, apresentando temperaturas moderadas, com verão quente e chuvoso. A temperatura média anual é de $21,2^{\circ} \mathrm{C}$; a média mensal máxima, de $27^{\circ} \mathrm{C}$; a média mensal mínima, de $14,2{ }^{\circ} \mathrm{C}$; e o índice pluviométrico médio anual, de $1.824 \mathrm{~mm}$ (Marques, 2003). O solo foi classificado como Latossolo Vermelho-Amarelo (Embrapa, 2006) textura argilosa (594 g kg-1 de argila, 224 de areia e 182 de silte). Antes da implantação das lavouras cafeeiras, há mais de 20 anos, a área era cultivada com culturas anuais de milho, feijão e pastagem. Para a implantação das lavouras cafeeiras em dezembro de 2003, foi realizado o preparo convencional do solo, com uma subsolagem a $0,40 \mathrm{~m}$, uma aração a 0,30 m, duas gradagens a $0,20 \mathrm{~m}$ e sulcamento a 0,40 $\mathrm{m}$ de profundidade. Os diferentes sistemas de manejo constituíram-se de:

Lavoura cafeeira com mecanização (CCM), cultivar Catuaí 2SL, implantada no espaçamento de $3,50 \times 0,70 \mathrm{~m}$. O controle de plantas invasoras foi realizado com roçadoras mecânicas, com média de três a quatro vezes ao ano, nas entrelinhas. Na linha de plantio foram realizadas duas aplicações manuais de herbicida pré-emergente no primeiro ano; a partir do segundo ano, as aplicações de herbicida foram em pósemergência, de forma mecanizada, duas vezes ao ano. Pulverizações com micronutrientes e defensivos agrícolas também foram mecanizadas duas vezes ao ano. Todos os implementos utilizados foram acoplados ao trator Massey Fergusson 275 (72 cv e massa de $4.393 \mathrm{~kg}$ ). Nesta lavoura, por estar em processo de formação, houve menos intensidade de tráfego de máquinas do que quando comparada a uma lavoura em processo de produção.

Lavoura cafeeira sem mecanização (CSM), cultivar Catuaí 2SL, implantada no espaçamento de $3,50 \times 0,70 \mathrm{~m}$. O controle de plantas invasoras foi feito com roçadeira costal nas entrelinhas e aplicação de herbicida pré-emergente com pulverizador costal 
na linha de plantio, no primeiro ano após o plantio. A partir do segundo ano, o herbicida pré-emergente foi substituído pelo de pós-emergência. As pulverizações com micronutrientes e defensivos foram realizadas com canhão (atomizador com seis bicos de $2.000 \mathrm{~L}$ ) acoplado ao trator, que percorre somente nos carreadores a cada $25 \mathrm{~m}$, e as adubações via solo foram realizadas manualmente.

Lavoura de café adensado (CA), cultivar Catuaí 2SL, espaçamento de 1,75 x 0,70 m. O controle de plantas invasoras foi feito com roçadeira costal nas entrelinhas e utilizando pulverizador costal, com herbicida de pré-emergência na linha de plantio no primeiro ano; a partir do segundo ano, foi utilizado somente herbicida em pós-emergência. As pulverizações com micronutrientes e defensivos agrícolas foram realizadas com atomizador com seis bicos de $2.000 \mathrm{~L}$ acoplado ao trator, que percorre somente nos carreadores a cada $25 \mathrm{~m}$, e as adubações via solo foram realizadas manualmente.

Mata nativa (MN) foi utilizada como referência, por ser uma área livre ou de mínimas intervenções antrópicas, o que mantém a estrutura do solo preservada.

As amostras indeformadas foram coletadas em agosto de 2007, com o auxílio de um amostrador de Uhland e anéis de alumínio de $6,35 \mathrm{~cm}$ de diâmetro por $2,54 \mathrm{~cm}$ de altura. As amostragens foram realizadas nas profundidades de $0-3 \mathrm{~cm}$ e $15-18 \mathrm{~cm}$, sendo esta última a camada de máxima resistência mecânica determinada previamente por penetrometria. As amostras foram retiradas em duas posições: na linha de tráfego e na projeção da copa dos cafeeiros, nos manejos CCM e CSM; e no centro das entrelinhas e na projeção da copa do cafeeiro, no manejo CA, com quatro repetições, na diagonal dos talhões. A amostragem na MN foi aleatória nas mesmas profundidades, totalizando 56 amostras: [(2 profundidades $\mathrm{x} 3$ manejos $\mathrm{x} 4$ repetições $\mathrm{x} 2$ posições) $+(2$ profundidades $\mathrm{x} 1$ manejo $\mathrm{x} 4$ repetições $\mathrm{x} 1$ posição)].

A densidade do solo foi determinada pelo método do cilindro de Uhland (Blake \& Hartge, 1986b) e com os excedentes dos anéis; a densidade de partículas foi determinada pelo método do picnômetro (Blake \& Hartge, 1986a), e a análise granulométrica, pelo método de Bouyoucus (Embrapa, 1997). Após a saturação das amostras indeformadas foram levadas para a unidade de sucção a $60 \mathrm{~cm}$ de coluna d'água, para obtenção do potencial matricial de $-6 \mathrm{kPa}$. Após a obtenção do equilíbrio, as amostras indeformadas foram levadas para o ensaio de resistência à penetração, utilizando um penetrógrafo de bancada marca Marconi ${ }^{\circledR}$ modelo MA-933. Para o ensaio de resistência à penetração, a haste com diâmetro da ponta de $4 \mathrm{~mm}$, área lateral do cone de $0,1968 \mathrm{~cm}^{2} \mathrm{e}$ ângulo de $45^{\circ}$ deslocou-se verticalmente a uma velocidade de $2 \mathrm{~mm} \mathrm{~s}^{-1}$. Em seguida, as amostras indeformadas foram colocadas em estufa a $105-110^{\circ} \mathrm{C}$ por $48 \mathrm{~h}$, para obtenção da massa de solo seco e posterior determinação da umidade gravimétrica (Embrapa, 1997).

A porosidade total foi obtida pela seguinte expressão: PT = [1 - (Ds/Dp)] x 100 (Vomocil, 1965). A microporosidade foi obtida pela determinação da umidade volumétrica de cada amostra correspondente ao potencial mátrico de - $6 \mathrm{kPa}$ (Embrapa, 1997), e a macroporosidade, pela diferença entre porosidade total e microporosidade. Todas as análises foram realizadas no Departamento de Ciências do Solo da Universidade Federal de Lavras. O experimento foi analisado usando delineamento com restrição na casualização, isto é, em parcela subsubdividida. A análise estatística foi realizada por meio do programa estatístico SISVAR (Ferreira, 2000), sendo os dados submetidos à análise de variância; a comparação das médias foi feita pelo teste de Scott-Knott a $5 \%$.

\section{RESULTADOS E DISCUSSÃO}

Os efeitos principais dos sistemas de manejo, posições, profundidades e as suas diferentes interações apresentaram resultado não significativo para a densidade do solo e o volume total de poros. Como se esperava haver diferença entre as posições nos sistemas de manejo, foi realizado o desdobramento da interação desses sistemas nas diferentes posições para esses atributos, que diferenciaram significativamente entre as posições amostradas e não entre os sistemas de manejo (Quadro 1), ao passo que nas profundidades não houve diferença. Esses resultados indicam que esses atributos não foram apropriados para quantificar o efeito do manejo sobre a estrutura do solo, corroborando Martins (2009), o que provavelmente pode ser explicado pelo curto tempo de estabelecimento da

Quadro 1. Propriedades físicas do solo sob diferentes sistemas de manejo, em diferentes posições analisadas: EL-linha de tráfego para os cafeeiros com e sem mecanização; centro das entrelinhas para o cafeeiro adensado; PC-projeção da copa para os cafeeiros; e aleatório para a mata nativa

\begin{tabular}{|c|c|c|c|c|}
\hline \multirow{2}{*}{ Manejo } & \multicolumn{2}{|c|}{ Ds } & \multicolumn{2}{|c|}{ VTP } \\
\hline & PC & EL & PC & EL \\
\hline & \multicolumn{2}{|c|}{$-\mathrm{Mg} \mathrm{m}^{-3}$} & \multicolumn{2}{|c|}{$-\mathrm{m}^{3} \mathrm{~m}^{-3}$} \\
\hline CCM & $1.09 \mathrm{bA}$ & $1.19 \mathrm{aA}$ & $0.56 \mathrm{aA}$ & $0.53 \mathrm{bA}$ \\
\hline CSM & $1.12 \mathrm{aA}$ & $1.10 \mathrm{aA}$ & $0.57 \mathrm{aA}$ & $0.57 \mathrm{aA}$ \\
\hline $\mathrm{CA}$ & $1.15 \mathrm{aA}$ & $1.16 \mathrm{aA}$ & $0.55 \mathrm{aA}$ & $0.54 \mathrm{aA}$ \\
\hline $\mathrm{MN}$ & $1.10 \mathrm{aA}$ & $1.10 \mathrm{aA}$ & $0.56 \mathrm{aA}$ & $0.56 \mathrm{aA}$ \\
\hline
\end{tabular}

Pares de média com mesma letra minúscula na linha e maiúscula na coluna não diferem entre si pelo teste de Skott-knott a $5 \%$. CCM: lavoura cafeeira com mecanização; CSM: lavoura cafeeira sem mecanização; CA: lavoura de café adensado; MN: mata nativa; Ds: densidade do solo; VTP: volume total de poros; PC: projeção da copa; EL: entrelinhas. 
cultura para ocasionar mudanças nesses atributos físicos do solo nos diferentes sistemas de manejo; no entanto, para a posição de linha de tráfego de máquinas esses atributos foram alterados. A densidade do solo e o volume total de poros mostraram-se significativamente maior e menor, respectivamente, na linha de tráfego de máquinas para o sistema de manejo CCM, em comparação com a mata nativa e os demais sistemas de manejo (CSM e CA). A mecanização aplicada em condições inadequadas pode comprometer as propriedades físicas do solo, como: densidade do solo e macroporosidade, conforme relatado por Oliveira et al. (2003), além de degradar a estrutura do solo, diminuindo o seu potencial produtivo (Silva et al., 2006).

$\mathrm{Na}$ interação entre os sistemas de manejo e as profundidades, houve alteração significativa para a macroporosidade, microposidade e relação de macro/ microporosidade, nos diferentes sistemas de manejo (Quadro 2).

O efeito das posições na macroporosidade mostrouse significativamente menor na posição de linha de tráfego para o sistema de manejo CCM, em comparação à projeção da copa, enquanto na comparação do efeito das profundidades ele se apresentou significativamente menor na profundidade de 0-3 cm, em relação à MN e aos demais sistemas de manejo (CSM e CA). Essas modificações causadas na estrutura do solo sob o sistema de manejo CCM devem-se possivelmente à pressão aplicada na superfície do solo pelo tráfego de máquinas e implementos agrícolas.

Na comparação dos diferentes sistemas de manejo nas diferentes posições, a macroporosidade mostrou- se significativamente menor nos sistemas de manejo CCM e CA na posição de linha de tráfego e entrelinhas (EL), respectivamente, ao passo que, na profundidade de 0-3 cm, para o sistema de manejo CCM, ela se apresentou significativamente menor, em comparação com a mata nativa e os demais sistemas de manejo. Os valores de macroporosidade para a posição de linha de tráfego, na profundidade de $0-3 \mathrm{~cm}\left(0.12 \mathrm{~m}^{3} \mathrm{~m}^{-3}\right)$, no sistema de manejo CCM, foram próximos do limite considerado ideal para o crescimento satisfatório do sistema radicular da maioria das plantas $\left(0.10 \mathrm{~m}^{3} \mathrm{~m}^{-3}\right)$, conforme relatado por Kiehl (1979) e Gupta \& Allmaras (1987). Os menores valores de macroporosidade na posição de linha de tráfego de máquinas e na profundidade de $0-3 \mathrm{~cm}$ para o sistema de manejo CCM devem-se possivelmente à pressão exercida pelo tráfego de máquinas e equipamentos agrícolas utilizado nesse sistema de manejo; também foi observado maior valor de microporosidade na profundidade de $0-3 \mathrm{~cm}$, refletindo a transformação dos macroporos em microporos.

A microporosidade não apresentou diferença significativa na comparação entre as posições, em nenhum sistema de manejo; contudo, a microporosidade apresentou-se significativamente menor na profundidade de $15-18 \mathrm{~cm}$, no sistema de manejo CCM, em comparação com a MN e os demais sistemas de manejo (CSM e CA). Na comparação dos sistemas de manejo nas diferentes posições, a microporosidade apresentou-se significativamente menor para a MN, em relação aos três sistemas de manejo (CCM, CSM e CA), e significativamente maior na profundidade de $0-3 \mathrm{~cm}$ para o sistema de manejo CCM, enquanto para a MN ela foi significativamente menor na profundidade de $15-18 \mathrm{~cm}$.

Quadro 2. Propriedades físicas do solo sob diferentes sistemas de manejo, em diferentes profundidades e posições analisadas: EL-linha de tráfego, para os cafeeiros com e sem mecanização; centro das entrelinhas, para o cafeeiro adensado; PC-projeção da copa, para os cafeeiros; e aleatório, para a mata nativa

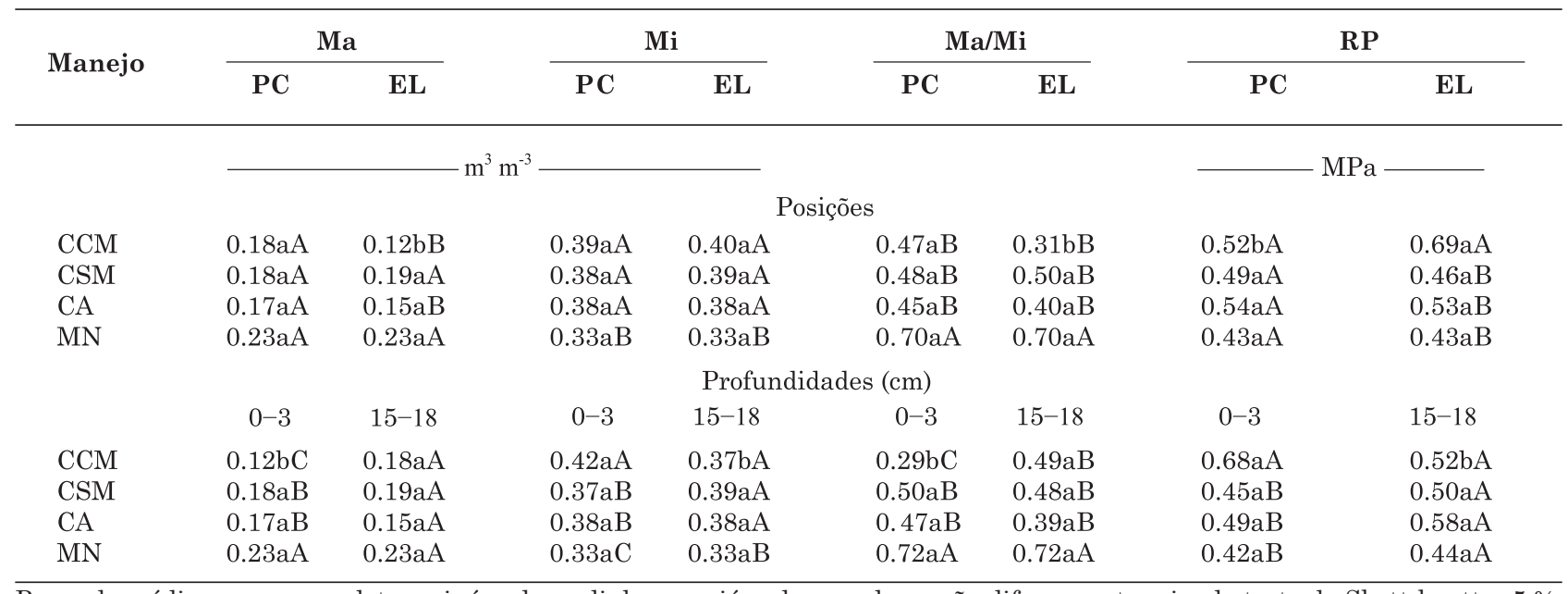

Pares de média com mesma letra minúscula na linha e maiúscula na coluna não diferem entre si pelo teste de Skott-knott a $5 \%$. CCM: lavoura cafeeira com mecanização; CSM: lavoura cafeeira sem mecanização; CA: lavoura de café adensado; MN: mata nativa; Ma: macroporosidade; Mi: microporosidade; PC: projeção da copa; EL: entrelinhas; RP: resistência à penetração. 
Comportamento semelhante foi observado por Gontijo et al. (2008) em um Latossolo Vermelho distrófico em Patrocínio, MG, avaliando diferentes posições na lavoura cafeeira (projeção da copa, linha de tráfego e centro da entrelinha). Nos Latossolos com baixa capacidade de retenção de água, a transformação de parte dos macroporos em microporos pode ser benéfica (Resende et al., 2007), por proporcionar maior retenção de água no solo.

$\mathrm{Na}$ comparação das posições, a relação de $\mathrm{Ma} / \mathrm{Mi}$ entre as posições analisadas foi significativamente menor na posição de linha de tráfego, enquanto nas profundidades ela apresentou-se significativamente menor na camada de $0-3 \mathrm{~cm}$ somente para o sistema de manejo CCM, em comparação com a MN e os demais sistemas de manejo com cafeeiros (CSM e CA). Avaliando o efeito dos sistemas de manejo em cada posição, verifica-se que a $\mathrm{MN}$ apresentou relação de $\mathrm{Ma} / \mathrm{Mi}$ significativamente maior, em comparação com os sistemas de manejo cultivado com cafeeiros (CCM, CSM e CA), nas duas posições (PC e EL). Esse resultado deve-se, possivelmente, ao preparo convencional antes da implantação das lavouras cafeeiras, que desagrega o solo e interfere na porosidade.

Comparando os sistemas de manejo em cada profundidade, verifica-se valor significativamente maior da relação $\mathrm{Ma} / \mathrm{Mi}$, na $\mathrm{MN}$, para as duas profundidades (0-3 e 15-18 cm), em comparação com os sistemas de manejo com cafeeiros (CCM, CSM e $\mathrm{CA}$ ), e significativamente menor na profundidade de 0-3 cm, para o manejo CCM, em comparação com MN, CSM e CA. De acordo com Kiehl (1979), um solo em condições ideais deve apresentar um terço da porosidade total formada por macroporos e os dois terços restantes por microporos, estabelecendo uma relação macroporos/microporos igual a 0,50. Com os resultados obtidos da relação $\mathrm{Ma} / \mathrm{Mi}$, verifica-se que esta, tanto nas profundidades quanto nos sistemas de manejo para a mata nativa e na posição de linha de tráfego e na profundidade de $0-3 \mathrm{~cm}$, para o sistema de manejo CCM, foi a que mais se distanciou do valor ideal $(0,50)$.

A resistência do solo à penetração apresentou-se significativamente maior na posição de linha de tráfego quando comparada à projeção da copa para o sistema de manejo CCM; na comparação entre os sistemas de manejo, ela mostrou-se significativamente maior no sistema de manejo CCM, para a posição de linha de tráfego de máquinas, em comparação com a $\mathrm{MN}$ e os demais sistemas de manejo (CSM e CA).

$\mathrm{Na}$ comparação entre as profundidades e entre os sistemas de manejo, a resistência do solo à penetração apresentou-se significativamente maior no sistema de manejo CCM, na profundidade de $0-3 \mathrm{~cm}$, quando comparado à $\mathrm{MN}$ e aos demais sistemas de manejo (CSM e CA), sendo os valores apresentados menores do que o considerado como crítico para o crescimento e desenvolvimento adequado das plantas, que é de 2,5 $\mathrm{MPa}$ (Canarache, 1990).
Algumas observações são necessárias para a correta avaliação da resistência do solo à penetração, entre as quais o fato de que esta é influenciada pela densidade e granulometria do solo e deve ser acompanhada pela umidade do solo (Dias Junior et al., 2004; Almeida et al., 2008), uma vez que o conteúdo de água no solo é o fator que governa a resistência mecânica e a quantidade de deformação que ocorre no solo (Dias Junior, 1994; Libardi \& Jong van Lier, 1999). Em solos com umidade muito baixa, a água encontra-se retida com maior tensão nos poros; a essa tensão somam-se as forças já existentes entre seus sólidos, fazendo com que, em solos com menor umidade, a resistência à deformação ou à penetração de raízes seja maior (Libardi \& Jong van Lier, 1999).

Além das alterações na macro e microporosidade, relação $\mathrm{Ma} / \mathrm{Mi}$ e resistência do solo à penetração, quatro anos pós-plantio, houve alterações visíveis no campo, que foram observadas pela ausência de cobertura vegetal e formação de encrostamento superficial em algumas áreas. Segundo Machado et al. (1981), essas alterações nas propriedades físicas do solo manifestam-se a partir do quarto ano e agravam-se após o oitavo ano consecutivo. Com isso, espera-se, com o decorrer do tempo, que haja interferência maior dos diferentes sistemas de manejo tanto na melhoria das propriedades físicas - em especial, no manejo adensado - quanto degradação no manejo com mecanização.

\section{CONCLUSÕES}

1. O sistema de café com mecanização alterou as propriedades físicas na posição de linha de tráfego de máquinas, indicadas pelo aumento da densidade do solo e resistência do solo à penetração e redução do volume total de poros, da macroporosidade e da relação macro/microporosidade, quatro anos após o plantio.

2. Comparando as profundidades, o sistema de café com mecanização mostrou menores valores de macroporosidade e relação macro/microposidade e maiores valores de microporosidade e resistência do solo à penetração, na profundidade de $0-3 \mathrm{~cm}$, quatro anos após o plantio.

3. As lavouras de café sob sistema de manejo adensado e sem mecanização preservaram, de modo geral, as propriedades físicas do solo no período de quatro anos pós-plantio.

\section{LITERATURA CITADA}

ANDREOLA, F.; COSTA, L.M. \& OLSZEVSKI, N. Influência da cobertura vegetal de inverno e da adubação orgânica e, ou, mineral sobre as propriedades físicas de uma Terra Roxa Estruturada. R. Bras. Ci. Solo, 24:857-865, 2000. 
ARSHAD, M.A.; LOWERY, B. \& GROSSMAN, B. Physical tests for monitoring soil quality. In: DORAN, J.W. \& JONES, A.J. Methods for assessing soil quality. Madison, Soil Science Society of America, 1996. p.123-141. (Special Publication, 49)

ALMEIDA, C.X.; CENTURION, J.F.; FREDDI, O.S.; JORGE, R.F. \& BARBOSA, J.C. Funções de pedotransferência para a curva de resistência do solo à penetração. R. Bras. Ci. Solo, 32:2235-2243, 2008.

BAYER, C. \& MIELNICZUK, J. Dinâmica e função da matéria orgânica. In: SANTOS, G.A.; SILVA, L.S.; CANELLAS, L.P. \& CAMARGO, F.A.O., eds. Fundamentos da matéria orgânica do solo em ecossistemas tropicais e subtropicais. 2.ed. Porto Alegre, Metrópole, 2008. p.7-18.

BAVER, L.D.; GARDNER, W.H. \& GARDNER, W.R. Soil physics. New York, J. Wiley, 1972. 498p.

BLAKE, G.R. \& HARTGE, K.H. Partycle density. In: KLUTE, A.. ed. Methods of soil analysis. 2.ed. Madison, American Society of Agronomy, 1986b. Part 1. p.377-382. (Agronomy Monography, 9)

BLAKE, G.R. \& HARTGE, K.H. Bulk density. In: KLUTE, A. ed. Methods of soil analysis. 2.ed. Madison, American Society of Agronomy, 1986a. Part 1. p.363-375.

COMPANHIA NACIONAL DE ABASTECIMENTO - CONAB. Disponível em: <http://www.conab.gov.br/conabweb/ index.php?PAG=73\&NSN=1217>. Acesso em: $18 \mathrm{de} \mathrm{dez.}$ 2009.

CARVALHO FILHO, A.; DA SILVA, R.P. \& FERNANDES, A.L.T. Compactação do solo em cafeicultura irrigada. Uberaba, Universidade de Uberaba, 2004. 44p. (Boletim Técnico, 3)

CANARACHE, A. PENETR - A generalized semi-empirical model estimating soil resistance to penetration. Soil Tillage Res., 16:51-70, 1990.

DAUDA, A. \& SAMARI, A. Cowpea yield response to soil compaction under trator on a sandy loam soil in the semiarid region of northern Nigeria. Soil Tillage Res., 68:17-22, 2002

DIAS JUNIOR, M.S. Compactação do solo. In: NOVAIS, R.F.; ALVAREZ V., H.V. \& SCHAEFER, C.E.G.R. Tópicos em ciência do solo. Viçosa MG, Sociedade Brasileira de Ciência do Solo, 2000. v.1. p.55-94.

DIAS JUNIOR, M.S. Compression of three soils under longterm tillage and wheel traffic. East Lansing, Michigan State University, 1994. 114p. (Tese de Doutorado)

DIAS JUNIOR, M.S.; SILVA, A.R.; FONSECA, S. \& LEITE, F.P. Método alternativo de avaliação da pressão de preconsolidação por meio de um penetrômetro. R. Bras. Ci. Solo, 28:805-810, 2004.

EMPRESA BRASILEIRA DE PESQUISA AGROPECUÁRIA . EMBRAPA. Centro Nacional de Pesquisas de Solos. Sistema brasileiro de classificação de solos. 2.ed. Rio de Janeiro, 2006. 306p.
EMPRESA BRASILEIRA DE PESQUISA AGROPECUÁRIA EMBRAPA. Centro Nacional de Pesquisas de Solos. Manual de métodos de análise de solo. 2.ed. Rio de Janeiro, 1997. $212 \mathrm{p}$

FERREIRA, D.F. Análises estatísticas por meio do SISVAR para Windows 4. 0. In: REUNIÃO ANUAL DA REGIÃO BRASILEIRA DA SOCIEDADE INTERNACIONAL DE BIOMETRIA, 45., 2000, São Carlos. Anais. São Carlos, Universidade Federal de São Carlos, 2000. p.255-258.

GONTIJO, I.; DIAS JUNIOR, M.S.; GUIMARÃES, P.T.G. \& ARAUJO-JUNIOR, C.F. Atributos físico-hídricos de um Latossolo de cerrado em diferentes posições de amostragem na lavoura cafeeira. R. Bras. Ci. Solo, 32:2227-2234, 2008.

GRANT, C.A. \& LAFOND, G.P. The effects of tillage systems and crop sequences on soil bulk density and penetration resistence on a clay soil in Southern Saskatchewan. Canadian J. Soil Sci., 73:223-232, 1993.

GUPTA, S.C. \& ALLMARAS, R.R. Models to access the susceptibility of soil to excessive compaction. Adv. Soil Sci., 6:65-100, 1987.

ISHAQ, M.; IBRAHIM, M.; HASSAN, A.; SAEED, M. \& LAL, R. Subsoil compaction effects on crops in Punjab, Pakistan: II. Root growth and nutrient uptake of wheat and sorghum. Soil Tillage Res., 60:153-161, 2001.

KARLEN, D.L. \& STOTT, D.E. A framework for evaluating physical, end chemical indicators of soil quality. In: DORAN, J.W.; COLEMAN, D.C.; BEZDICEK, D.F. \& STEWART, B.A. eds. Defining soil quality for a sustainable environment. Madison, SSSA, 1994. p.53-71. (Special. Publication, 35)

KIEHL, E.J. Manual de edafologia. São Paulo, Ceres, 1979. 262 .

LIBARDI, P.L \& JONG van LIER, Q. Atuação dos fatores físicos do solo no desenvolvimento do sistema radicular. In: FERNANDES, M.F.; TAVARES, E.D. \& LEAL, M.L., eds. WORKSHOP SOBRE SISTEMA RADICULAR: Metodologias e estudo de casos. Aracaju, Embrapa Tabuleiros Costeiros, 1999. p.47-56.

MIRANDA, E.E.V.; DIAS JUNIOR, M.S.; GUIMARÃES, P.T.G.; PINTO, J.A.O.; ARAÚJO JUNIOR, C.F. \& LASMAR JUNIOR, E. Efeito do manejo e do tráfego nos modelos de sustentabilidade da estrutura de um Latossolo Vermelho cultivado com cafeeiros. Ci. Agrotec., 1506-1515, 2003. (Edição especial)

MARQUES, H.S. Uso de geotecnologias no estudo das relações entre solos, orientação de vertentes e o comportamento espectral de áreas cafeeiras em Machado, Minas Gerais. Lavras, Universidade Federal de Lavras, 2003. 82p. (Tese de Mestrado)

MARTINS, P.C.C. Avaliação da sustentabilidade da estrutura de um Cambissolo sob difetentes sistemas de uso da terra na Amazônia Ocidental. Lavras, Universidade Federal de Lavras, 2009. 55p. (Tese de Mestrado) 
MACHADO, J.A.; SOUZA, D.M.P. \& BRUM, A.C.R. Efeito de anos de cultivo convencional em propriedades físicas do solo. R. Bras. Ci. Solo, 5:187-189, 1981.

NOVAK, L.R.; MANTOVANI, E.C.; MARTYN, P.J. \& FERNADES, B. Efeito do tráfego de trator e da pressão de contato pneu/solo na compactação de um Latossolo Vermelho-Escuro álico, em dois níveis de umidade. Pesq. Agropec. Bras., 27:1587-1595, 1992.

OLIVEIRA, G.C.; DIAS JUNIOR, M.S.; RESCK, D.V.S. \& CURI, $\mathrm{N}$. Alterações estruturais e comportamento compressivo de um Latossolo Vermelho distrófico argiloso sob diferentes sistemas de uso e manejo. Pesq. Agropec. Bras., 38:47-50, 2003

PAVAN, M.A.; CHAVES, J.C.D.; SIQUEIRA, R.; ANDROCIOLI FILHO, A.; COLOZZI FILHO, A. \& BALOTA, E.L. High coffee population density to improve fertility of an Oxisol. Pesq. Agropec. Bras., 34:459-465, 1999.

PAVAN, M.A. \& CHAVES, J.C.D. Influência da densidade de plantio de cafeeiros sobre a fertilidade do solo. In: SIMPÓSIO INTERNACIONAL SOBRE CAFÉ ADENSADO, 1996, Londrina. Anais... Londrina, IAPAR, 1996. p.87-105.

RESENDE, M.; CURI, N.; REZENDE, S.R. \& CORRÊA, G.F. Pedologia: Base para distinção de ambientes. 5.ed. Lavras, Universidade Federal de Lavras, 2007. 322p.

SILVA, A.P.; LIBARDI, P.L. \& CAMARGO, O.A. Influência da compactação nas propriedades físicas de dois Latossolos. R. Bras. Ci. Solo, 10:91-95, 1986.
SILVA, R.V.; REINERT, D.J.; REICHERT, J.M. \& SOARES, J.M. Fatores controladores da compressibilidade de um Argissolo Vermelho-Amarelo distrófico arênico e de um Latossolo Vermelho distrófico típico. I - Estado inicial de compactação. R. Bras. Ci. Solo, 26:1-8, 2002.

SILVA, R.B.; DIAS JUNIOR, M.S.; SILVA, F.A.M. \& FOLE, S.M. O tráfego de máquinas agrícolas e as propriedades físicas, hídricas e mecânicas de um Latossolo dos Cerrados. R. Bras. Ci. Solo, 27:973-983, 2003.

SILVA, A.R.; DIAS JUNIOR, M.S.; GUIMARÃES, P.T.G. \& ARAUJO JUNIOR, C.F. Modelagem da capacidade de suporte de carga e quantificação dos efeitos das operações mecanizadas em um Latossolo Amarelo cultivado com cafeeiros. R. Bras. Ci. Solo, 30:207-216, 2006.

SECCO, D.; REINERT, D.J.; REICHERT, J.M. \& ROS, C.O. Produtividade de soja e propriedades físicas de um Latossolo submetido a sistemas de manejo e compactação. R. Bras. Ci. Solo, 28:797-804, 2004.

TORMENA, C.A. \& ROLLOF, G. Dinâmica da resistência à penetração de um solo sob plantio direto. R. Bras. Ci. Solo, 20:333-339, 1996.

TORRES, E. \& SARAIVA, O.F. Camadas de impedimento mecânico do solo em sistema agrícolas com soja. Londrina, Embrapa-CNPSoja, 1999. 58p. (Circular Técnica, 23)

VOMOCIL, J.A. Porosity. In: BLAKE, C.A. Methods of soil analysis. Madison, American Society of Agronomy, 1965. 